\title{
Success of Bubble CPAP in Treatment of Respiratory Distress Syndrome in Preterm Infants
}

Tufail Soomro $^{1^{*}}$ and Shiyam Sunder Tikmani ${ }^{2}$

${ }^{1}$ Department of Pediatrics, Ghulam Mohammad Mahar Medial College, Sukkur, Pakistan

${ }^{2}$ Aga Khan University, Department of Community Health Sciences, Karachi, Pakistan

*Corresponding author: Tufail Soomro, Department of Pediatrics, Ghulam Mohammad Mahar Medial College, Sukkur, Pakistan, Tel: +923337279661; E-mail: drtufailsoomro@hotmail.com

Received date: July 18, 2016, Accepted date: August 30, 2016, Published date: August 31, 2016

Copyright: ( 2016 Soomro T, et al. This is an open-access article distributed under the terms of the Creative Commons Attribution License, which permits unrestricted use, distribution, and reproduction in any medium, provided the original author and source are credited.

\begin{abstract}
Background: To observe the survival of preterm infants with respiratory distress syndrome treated with bubble CPAP in a tertiary care hospital.

Methods: This prospective observational study was conducted in the NICU of Civil hospital Sukkur from $1^{\text {st }}$ July 2013 to $18^{\text {th }}$ August 2015 . All consecutively born preterm neonates with gestational age between 27 weeks to 36 weeks of gestation, admitted to the neonatal intensive care unit with respiratory distress are included in the study. These neonates were managed by Bubble continuous positive airway pressure (bCPAP) in combination with surfactant (bovine derivative) via intubation, Surfactant replacement, extubation (INSURE) protocol Success was considered when neonates were clinically stable and required $<0.3 \mathrm{FiO}_{2}$ to maintain oxygen Saturation $>90 \%$ and $\mathrm{PaCO}_{2}<40-45 \mathrm{mmHg}$.
\end{abstract}

Results: Mean age of enrolled infants was $1.35 \pm 0.60$ days, $70(57.9 \%)$ were males and $51(42.1 \%)$ were female, mean weight was $1.76 \pm 0.37 \mathrm{~kg}$ and mean gestational age was $33 \pm 2.3$ weeks. Overall $77(63.6 \%)$ preterm infants were successfully weaned off from CPAP. Multivariable analysis showed weight of infant less than $1.5 \mathrm{~kg}$ or 8.63 (95\% Cl: $1.71-43.57)$, respiratory rate of more than 70 breaths per minute, or 9.59 (95\% Cl: 2.59-35.52), nasal flaring or 3.35 (95\% Cl: 1.08-10.31) and typical findings of RDS on chest X-rays 12.04 (95\% Cl: 1.89-76.52) are independent risk factors for failure of bCPAP. However, gestational age 0.70 ( $95 \% \mathrm{Cl}$ : $0.54-0.89)$ is protective against failure.

Conclusion: bCPAP has a high proportion of success in the management of respiratory distress syndrome in preterm. It could be most useful in resource-constrained settings.

Keywords: Respiratory distress; Preterm infants

\section{Introduction}

Annually about 15 million preterm babies are born around the world and more than one million die soon after birth mainly due to respiratory complications [1,2]. Respiratory distress syndrome (RDS) is a common and serious complication of preterm birth accounting for $50 \%$ cases $[3,4]$ preterm, Therefore, it is the major cause of mortality in preterm neonates [5]. Recent advances in the perinatal care have led to the markedly improved survival of preterm infants during the last two decades. Conventionally neonates with respiratory distress are managed by respiratory support with positive pressure ventilation (delivered usually by mechanical ventilator) and surfactant replacement therapy [6]. Surfactant replacement via intubation, surfactant replacement, and extubation to CPAP (INSURE) protocol described by Verder et al. [7] in 1994, remarkably reduced the need for mechanical ventilator from $85 \%$ to $41 \%$ in the preterm neonate. In the developed world mechanical ventilator and CPAP machines are the mainstays of respiratory support in neonates [6] but these machines are too expensive and many resources constrained, low socioeconomic countries [6]. The effectiveness of locally adapted bCPAP has been documented $[8,9]$. bCPAP is a simple and cost effective respiratory support system (RSS) which consists of products that are easily available and health care provider can easily be trained to make and use this RSS [8]. bCPAP circuits consisted of inspiratory limb, the interface (nasal prongs) and the proximal part of the expiratory limb. The proximal end of the inspiratory limb connects the humidified oxygen source (wall piped oxygen) through the interface to the baby [8]. These tubes are carefully secured with an adhesive plaster to ensure that the length immersed in water remains constant [8]. The bCPAP generator is a cylindrical, transparent bottle filled to predetermined level with distilled water. The expiratory limb of the circuit is immersed in this bottle and the depth of the immersion in centimeters below the water surface correspond to the desired bCPAP in $\mathrm{cm}_{2} \mathrm{O}$ usually between $5 \mathrm{~cm}$ to $8 \mathrm{~cm}$ of $\mathrm{H}_{2} \mathrm{O}$ [8]. This provides positive pressure in the whole respiratory cycle [9], increases the functional residual capacity of lungs and lowers work of breathing [10]. Ultimately, bCPAP reduces the need for mechanical ventilation, morbidity, mortality [6-11]. Many studies have been shown that locally manufactured bCPAP system showed promising results $[9,12]$. There is a paucity of studies on the effectiveness of bCPAP in Pakistan. In this study, we intend to observe the efficacy of Bubble CPAP with surfactant replacement therapy in the management of RDS in preterm neonates. 


\section{Materials and Methods}

This prospective observational study was conducted in the neonatal intensive care unit (NICU) of Civil hospital Sukkur from $1^{\text {st }}$ July 2013 to $18^{\text {th }}$ August 2015. All consecutively born preterm neonates with gestational age between 27 weeks to 36 weeks admitted with respiratory distress have a characteristic chest radiographic appearance i.e. uniform reticulogranular pattern with or without air bronchogram, an arterial oxygen tension $\left(\mathrm{PaO}_{2}\right)<50 \mathrm{mmHg}$ and a requirement for supplemental oxygen to maintain oxygen saturation over $85 \%$ were included in this study. Infants with a gestational age more than 36 weeks or requiring mechanical ventilation at the time of admission or having major congenital malformation including congenital diaphragmatic hernia, trachea-esophageal fistula, cleft lip/palate; chromosomal abnormalities such as trisomy [13-18], significant morbidity besides RDS including cardiac diseases; parent refused to participate in the study were excluded. Eligible infants were started on bCPAP with bi-nasal prongs. Positive end-expiratory pressure (PEEP) was started at $5 \mathrm{~cm}$ of $\mathrm{H}_{2} \mathrm{O}$ and was adjusted to minimized chest retraction. $\mathrm{FiO}_{2}$ was adjusted to maintain $\mathrm{SpO}_{2} 88 \%$ to $94 \%$. Flow was titrated to produce continuous bubbling in the chamber. Surfactant (bovine derivative) was administered via intubation, surfactant replacement, extubation (INSURE) protocol. If Fraction of Inspired Oxygen $\left(\mathrm{FiO}_{2}\right)$ requirement $\geq 0.4$ then a dose of surfactant repeated after $6 \mathrm{~h}$ to $12 \mathrm{~h}$ of the first dose for maximum three doses. bCPAP was considered to be successful if neonate is clinically stable, respiratory distress improved and the baby could be successfully weaned off from bCPAP. Preterm infants with no subcostal recession, no nasal flaring, the respiratory rate between 50 per min and 60 per min and $\mathrm{O}_{2}$ saturations of $>90 \%$ on $\mathrm{FiO}_{2}<30 \%$ and PEEP $<5 \mathrm{~cm}$ of water were weaned off from bCPAP. Neonates were labeled as having CPAP failure and were started on mechanical ventilator when they remained hypoxic i.e. estimated arterial oxygen saturation $\left(\mathrm{SpO}_{2}\right)<87 \%$ despite
$\mathrm{FiO}_{2}>70 \%$ and PEEP $>7 \mathrm{~cm}$ of $\mathrm{H}_{2} \mathrm{O}$; severe retraction on PEEP $>7 \mathrm{~cm}$ of $\mathrm{H}_{2} \mathrm{O}$; prolong ( $>20$ seconds) and recurrent apnea and partial pressure of carbon dioxide $\left(\mathrm{PaCO}_{2}\right)>60 \mathrm{mmHg}$ with $\mathrm{pH}<7.2$ on two consecutive arterial blood analysis within $30 \mathrm{~min}$.

Data on age, birth weight, gestational age, gender, respiratory rate, heart rate, nasal flaring, subcostal recession, typical findings of RDS on chest X-ray chest were recorded by a doctor on pre-approved proforma.

By taking a proportion of success of bCPAP $75 \%$ with a margin of error $8 \%$ at $95 \%$ confidence interval the calculated sample size was 121 preterm neonates by using World Health Organization (WHO) software for sample size calculation [14]. Data was entered and analyzed using SSPS (SPSS Inc. Chicago, IL, USA) [18]. Mean and standard deviation computed for quantitative variables including age, birth weight, gestational age, respiratory rate and heart rate. Frequencies and percentages were reported for categorical variables including gender, nasal flaring, subcostal recession and chest X-ray showing signs of RDS. Student t-test was used to compare continuous variables and Chi-Square test applied to compare categorical variables. A p-value of $<0.05$ will be taken as significant. Multivariable logistic regression analysis was done to determine factors of failure of infants treated with bCPAP. Model fit was assessed through Hosmer and Lemeshow test. This study was approved by hospital ethical review committee.

\section{Results}

Total 121 preterm infants were enrolled in the study. Mean age of enrolled infants was $1.35 \pm 0.60$ days, 70 (57.9\%) were males and 51 (42.1\%) were female, mean weight was $1.76 \mathrm{~kg} \pm 0.37 \mathrm{~kg}$ and mean gestational age was $33 \pm 2.3$ weeks (Table 1 ).

\begin{tabular}{|c|c|c|c|c|}
\hline & Total & Failure & Success & \multirow[t]{2}{*}{ P-value } \\
\hline & $(n=121)$ & $(n=44)$ & $(n=77)$ & \\
\hline Mean age (in days) & $1.35 \pm 0.60$ & $1.32 \pm 0.6$ & $1.36 \pm 0.6$ & 0.691 \\
\hline \multicolumn{5}{|l|}{ Gender } \\
\hline Male & $70(57.9 \%)$ & $24(54.5 \%)$ & $46(59.7 \%)$ & 0.357 \\
\hline Female & $51(42.1 \%)$ & $20(45.5 \%)$ & $31(40.3 \%)$ & \\
\hline Mean weight (kg) & $1.76 \pm 0.37$ & $1.56 \pm 2.8$ & $1.86 \pm 0.38$ & $<0.001$ \\
\hline Mean gestational age (weeks) & $33 \pm 2.3$ & $33.57 \pm 2.5$ & $32.68 \pm 2.18$ & 0.43 \\
\hline Mean respiratory rate $(\mathrm{b} / \mathrm{min})$ & $64 \pm 4.9$ & $65 \pm 5.2$ & $61.86 \pm 4.02$ & $<0.001$ \\
\hline Respiratory rate $>70$ & $76(62.8 \%)$ & $38(86.4 \%)$ & $38(49.4 \%)$ & $<0.001$ \\
\hline Mean heart rate $(\mathrm{b} / \mathrm{min})$ & $159.35 \pm 8.3$ & $159.11 \pm 9.62$ & $159.48 \pm 7.62$ & 0.818 \\
\hline Nasal flaring & $71(58.7 \%)$ & $32(72.7 \%)$ & $39(50.6 \%)$ & 0.014 \\
\hline Subcostal recession & $96(79.3 \%)$ & $41(93.2 \%)$ & $55(71.4 \%)$ & 0.003 \\
\hline Typical finding of RDS on chest X-rays & $81(66.9 \%)$ & $42(95.5 \%)$ & $39(50.6 \%)$ & $<0.001$ \\
\hline
\end{tabular}

Table 1: Baseline characteristics of infants. 


\begin{tabular}{|c|c|c|}
\hline & Crude or $(95 \% \mathrm{Cl})$ & Adjusted or $(95 \% \mathrm{Cl})$ \\
\hline Mean age (in days) & $1.14(0.61-2.13)$ & $1.41(0.54-3.67)$ \\
\hline \multicolumn{3}{|l|}{ Gender } \\
\hline Female & 1 & 1 \\
\hline Male & $0.81(0.38-1.71)$ & $0.71(0.23-2.11)$ \\
\hline Weight of infant $<1.5 \mathrm{~kg}$ & $7.42(2.48-22.12)$ & $8.63(1.71-43.57)$ \\
\hline Mean gestational age (weeks) & $0.84(0.71-0.99)$ & $0.70(0.54-0.89)$ \\
\hline Respiratory rate $>70$ & $6.5(2.46-17.14)$ & $9.59(2.59-35.52)$ \\
\hline Mean heart rate $(\mathrm{b} / \mathrm{min})$ & $1.01(0.96-1.05)$ & $1.02(0.95-1.09)$ \\
\hline Nasal flaring & $2.59(1.17-5.78)$ & $3.35(1.08-10.31)$ \\
\hline Subcostal recession & $5.46(1.53-19.51)$ & $3.87(0.54-27.40)$ \\
\hline Typical finding of RDS on chest X-rays & $20.46(4.62-90.53)$ & $12.04(1.89-76.52)$ \\
\hline
\end{tabular}

Table 2: Predictors of treatment failure among infants on bubble CPAP.

Of 121 infants, mean respiratory rate was $64 \pm 4.9$ breaths per min, $76(62.8 \%)$ has respiratory rate of more than 70 breaths per min; mean heart rate $159.35 \pm 8.3$ beats per minutes; $71(58.7 \%)$ had nasal flaring, $96(79.3 \%)$ has subcostal recession and $81(66.9 \%)$ had typical X-ray findings of RDS.

Overall 77 (63.6\%) preterm infants were successfully weaned off from bCPAP. Comparative analysis of preterm infants who successfully wean off and who fail bCPAP is summarized above in (Table 2).

Univariable analysis showed the weight of the infant, gestational age, respiratory rate of more than 70 , nasal flaring, subcostal recession and typical findings of RDS on chest X-ray are factors associated with failure of bCPAP. Multivariable analysis showed weight of infant less than $1.5 \mathrm{~kg}$ OR 8.63 (95\% CI: 1.71-43.57), respiratory rate of more than 70 breaths per minute, or 9.59 (95\% CI: 2.59-35.52), nasal flaring or 3.35 (95\% CI: 1.08-10.31) and typical findings of RDS on chest X-rays 12.04 (95\% CI: 1.89-76.52) are independent risk factors for failure of bCPAP. However, gestational age 0.70 (95\% CI: 0.54-0.89) is protective against failure.

\section{Discussion}

We evaluated the effectiveness of bCPAP in the management of RDS in the preterm neonate. The role of Bubble CPAP in the management of RDS was emphasized by many researchers. Randomized controlled trials also conducted in India and Australia in which they compared the safety profile and effectiveness of bubble CPAP with a mechanical ventilator in preterm infants. Neonates treated with Bubble CPAP were more successful than mechanical ventilator [8]. Lee et al. [18] also elicited the superiority of bCPAP in preterm neonates in comparison to a mechanical ventilator. They also emphasized on the usefulness of bCPAP in very low birth neonates [13]. In our study, only 44 neonates out of 121 who were started on Bubble CPAP require mechanical ventilation. Only 3 babies had pneumothorax which was successfully managed. In a retrospective study by Ammari et al. [15], the failure rate of bCPAP was $24 \%$ in babies $<1250 \mathrm{~g}$ and $50 \%$ in babies $<750 \mathrm{~g}$. None of the baby $>30$ weeks gestation failed Bubble CPAP. The difference between success rate with our study is due to we made bubble CPAP which is most economical (manually device) while in the study by Ammari et al. [15] used Fischer and Paykel bubble CPAP. Another reason could be inadequate infection control in public sector hospital. In a case-control study by Boo et al. [16] of the 97 preterm babies with RDS on ventilator CPAP or bubble CPAP, 38\% failed CPAP and require mechanical ventilation [16].

Results of our study showed weight of infant less than $1.5 \mathrm{~kg}$ or 8.63 (95\% CI: 1.71-43.57), respiratory rate of more than 70 breaths per min, or 9.59 (95\% CI: 2.59-35.52), nasal flaring OR 3.35 (95\% CI: 1.08-10.31) and typical findings of RDS on chest X-rays 12.04 (95\% CI: 1.89-76.52) are independent risk factors for failure of bubble CPAP. However, gestational age 0.70 (95\% CI: 0.54-0.89) is protective against failure. Ammari et el. [15] reported typical findings of RDS on the chest $\mathrm{x}$-ray was an important predictor of bubble CPAP failure. In other uncontrolled studies and in the studies comparing INSURE with ventilation, CPAP failure rate ranges from $14 \%$ to $40 \%$. Koti et al. [14] conduct a retrospective analytical study on 56 neonates (28 weeks to 34 weeks) reported the failure of bubble CPAP of $25 \%$.

The higher incidence of sepsis in our study is a lack of proper facilities and trained staff for infection control. The higher rate of CPAP failure in the study of Boo et al. [16] may be attributed to the lesser use of surfactant. These differences are mainly due to the paramedical care provided to the neonates and lesser incidence of septicemia.

There are some limitations to the study. First, we did not account for infections in these infants. However, blood cultures were negative in all these infants. Second, this is a single-center study so results cannot be generalized.

In conclusion, RDS is a potentially treatable problem of all preterm infants. It can be treated by a mechanical ventilator or bCPAP along 
Citation: Soomro T, Tikmani SS (2016) Success of Bubble CPAP in Treatment of Respiratory Distress Syndrome in Preterm Infants . J Gen Pract

Page 4 of 4

with surfactant replacement. In this study, we observe the success rates of locally manufactured bubble CPAP in the management of RDS to reduce morbidity, mortality and economic burden of this disease.

\section{References}

1. Howson CP, Kinnay MV, Lawn JE (2012) March of Dimes, PMNCH, Save the Children, WHO. Born Too Soon: The Global Action Report on Preterm Birth, World Health Organization, Geneva.

2. Hussain S (2012) Pakistan stands fourth globally in preterm births: report. Today's Newspaper.

3. Gnanaratnem J, Finer NN (2000) Neonatal acute respiratory failure. Curr Opin Pediatr 12: 227-232.

4. St Clair C, Norwitz ER, Woensdregt K, Cackovic M, Shaw JA, et al. (2008) The probability of neonatal respiratory distress syndrome as a function of gestational age and lecithin/sphingomyelin ratio. Am J Perinatol 25: 473-480.

5. Kawaza K, Machen HE, Brown J, Mwanza Z, Iniguez S, et al. (2014) Efficacy of a low-cost bubble CPAP system in the treatment of respiratory distress in a neonatal ward in Malawi. PLoS One 29: e86327.

6. Kamath BD, Mac Guire ER, Mc Clure EM, Goldenberg RL, Jobe AH (2011) Neonatal Mortality from Respiratory Distress Syndrome: Lessons for Low-Resource Countries. Pediatrics 127: 1139-1146.

7. Verder H, Robertson B, Greisen G, Ebbesen F, Albertsen P, et al. (1994) Surfactant therapy and nasal continuous positive airway pressure for newborn with respiratory distress syndrome. $\mathrm{N}$ Engl J Med 331: 1051-1055.

8. Kaur C, Sema A, Beri RS, Puliyel JM (2008) A simple circuit to deliver Bubble CPAP. Indian Pediatrics 45: 312-314.

9. Chan KM, Chan HB (2007) The use of Bubble CPAP in preterm infants: local experience. HK J Paediatr 12: 86-92.
10. Courtney SE, Pyon KH, Saslow JG, Arnold GK, Pandit PB, et al. (2001) Lung recruitment and breathing pattern during variable versus continuous flow nasal continuous positive airway pressure in preterm infants: an evaluation of three devices. Pediatrics 107: 304-308.

11. Diblasi RM (2009) Nasal Continuous Positive Airway Pressure (CPAP) for the Respiratory Care of the Newborn Infant. Respir Care 54: 1209-1235.

12. Koyamaibole L, Kado J, Qovu JD, Colquhoun S, Duke T, et al. (2006) An evaluation of bubble-CPAP in a neonatal unit in a developing country: effective respiratory support that can be applied by nurses. J Trop Pediatr 52: 249-53.

13. Nowadzky T, Pantoja A, Britton JR (2009) Bubble Continuous Positive Airway Pressure, A potentially better practice, reduces the use of mechanical ventilation among very low birth weight infants with respiratory distress syndrome. Pediatrics 123: 1534-1540.

14. Koti J, Murki S, Gaddam P, Reddy A, Reddy MD (2010) Bubble CPAP for respiratory distress syndrome in preterm infants. Indian Pediatr 47: 139-143.

15. Ammari A, Suri M, Milisavljevic V, Sahni R, Bateman D, et al. (2005) Variables associated with the early failure of nasal CPAP in very low birth weight infants. J Pediatr 147: 341-347.

16. Boo NY, Zuaidah AL, Lim NL, Zulfiqar MA (2000) Predictors of failure of nasal continuous positive airway pressure in the treatment of a preterm infant with respiratory distress syndrome. J Trop Pediatr 46: 172-175.

17. Tagre A, Kadam S, Vaidya U, Pandit A, Patole S (2013) A pilot study of Comparison of BCPAP vs. VCPAP in preterm infants with early onset Respiratory distress. J Trop Pediatr 59: 113-119.

18. Lee KS, Dunn MS, Fenwick M, Shennan AT (1988) A comparison of underwater bubble continuous positive airway pressure with ventilatorderived continuous positive airway pressure in preterm neonates ready for extubation. Biol Neonate 73: 69-75. 